\title{
Coupled electrical-thermal model for monopolar and bipolar radiofrequency liver tumor ablation
}

\author{
Frederik Soetaert, Guillaume Crevecoeur, and Luc Dupré
}

\begin{abstract}
Radiofrequency ablation (RFA) is an alternative cancer treatment that applies radiofrequency electric fields to biological tissues. The subsequent electrical currents induce Joule heating and inflict local thermal damage to the biological tissue. Previous clinical studies demonstrate the possibilities and benefits of applying RFA for the treatment of liver tumors. Currently, most clinical studies focus on monopolar RFA, consisting of a single electrode and a grounding pad, and its therapeutical outcome. This study however focuses on a bipolar configuration where two needle electrodes are inserted at a certain potential difference. In order to assess the temperature elevations in biological tissues, we have developed a computational model. A mathematical model based on Laplace's equation (with appropriate boundary conditions) is used to model the electromagnetic phenomena and is coupled to the bioheat transfer equation. This study compares a monopolar RFA with a bipolar configuration. We investigate the spatio-temporal temperature variations using detailed numerical three-dimensional finite element simulations in biological tissues, corresponding to liver tissue. Results show that the ablated region in between the two needles of the bipolar configuration can be enlarged compared to a monopolar RFA configuration.
\end{abstract}

Index Terms-radiofrequency ablation, electrical-thermal problems, coupled problems, thermotherapy, simulation, numerical techniques

\section{INTRODUCTION}

$\mathbf{R}$ ADIOFREQUENCY electric fields can locally heat tumoral tissues. This basic mechanism underlying radiofrequency ablation (RFA) is utilized as a promising cancer treatment technique. RFA has been extensively studied and considered in various clinical studies. The efficacy of RFA for the treatment of patients with liver tumors has for instance been reported in e.g. [1] and more recently in [2]. Liver cancer is the fourth most common cancer worldwide. Unfortunately, only a small proportion of patients can benefit from the traditional treatment combinations (surgery, chemotherapy and radiotherapy). Patient studies confirm that the treatment of small tumors with RFA is superior compared to surgery [3]. Tumor sizes with larger volumetric dimensions (larger than 2$3 \mathrm{~cm}$ ) are more difficult to treat using RFA [4]. Besides the extensive studies and applications for liver tumors, RFA has also been applied as cancer treatment modality in other organs such as kidney [5], bone [6], lungs [7], etc.

Computational models have been developed as a tool to understand RFA's working principle and to increase its efficacy [8], [9], [10]. In some RFA applications, a part of the tumor cells often survive because the temperature in some regions

F. Soetaert is a Ph.D. fellow of the Research Foundation - Flanders (FWO).

F. Soetaert, G. Crevecoeur, L. Dupré are with the Department of Electrical Energy, Systems and Automation, Ghent University, Ghent, Belgium, e-mail: frederik.soetaert@ugent.be

978-1-4673-9575-5/16/\$31.00 @2016 IEEE does not exceed the therapeutic limit $\left(41-42^{\circ} \mathrm{C}\right)$ [11], leading to high recurrence rates of the tumors. The dynamics of the thermal processes need to be calculated to assess whether complete tumor necrosis can be achieved by means of RFA. The mathematical modeling relies on the coupling of an electromagnetic heat source and a model of the temperature dynamics in the biological tissue. Simulations of the coupled electrical-thermal effects in RFA can be performed by means of numerical finite element methods [12] or finite difference methods [13].

The traditional RFA configuration consists of a single needle and a grounding pad. Besides this monopolar RFA configuration, the use of two needles has recently been considered in a bipolar configuration for cardiac ablation studies [14]. The authors simulated in [13] a bipolar configuration for liver tumor ablation where 2 needles inject pulsed radiofrequency currents using the finite difference method. Here, we apply detailed 3D finite element methods to compare the bipolar and monopolar configuration for radiofrequency liver tumor ablation. We place the needles at a certain voltage and compare the spatio-temporal distribution to the one of a monopolar RFA configuration. The survival rate of the biological tissue can be calculated using a tissue damage model. The aim of our study is to compare the spatial extent of the elevated temperatures when applying monopolar and bipolar RFA.

\section{Methods}

\section{A. Coupled electrical-thermal model}

Biological tissues can be considered as quasi-static in the radiofrequency range. The contribution of the dielectric permittivity to the impedance can therefore be neglected and the biological tissue can be treated as a resistive material. A certain electric field $\mathbf{E}$ induces a current density $\mathbf{J}$ at coordinate $\mathbf{r}$ in the tissue and is described by Ohm's law:

$$
\mathbf{J}(\mathbf{r})=\sigma \mathbf{E}(\mathbf{r})
$$

with electrical conductivity $\sigma$. The spatial distribution of the electric field $\mathbf{E}(\mathbf{r})=-\nabla V(\mathbf{r})$ and its associated electric scalar potential $V$ can be calculated using Laplace's equation

$$
\nabla \cdot[\sigma \nabla V(\mathbf{r})]=0
$$

The appropriate boundary conditions need to be assigned to the Laplace's equation and have to encompass the application of radiofrequency ablation by fixing a value of the electric potential to the boundary of the needles. This electric potential at the needle surface can be time dependent $V_{n}(t)$. The electric 
fields and induced currents result in resistive heating $Q(\mathbf{r})$ by Joule's law:

$$
Q(\mathbf{r})=\mathbf{J}(\mathbf{r}) \mathbf{E}(\mathbf{r})=\sigma|E(\mathbf{r})|^{2}
$$

Equation (3) acts as a power source term that will drive the heat transfer. Three mechanisms can transfer heat from differences in temperature: convection, conduction and radiation. The latter can be disregarded for the application of radiofrequency ablation. The Pennes' bioheat equation expresses heat transfer in biological tissues [15]. This partial differential equation of the time- and space-dependent temperature $T$ contains the diffusion of heat $\nabla \cdot(k \nabla T)$, a cooling term $m\left(T-T_{b}\right)$ due to local blood perfusion at temperature $T_{b}$, and the electromagnetic heat source (3). The $m\left(T-T_{b}\right)$ can model the heat transfer in the capillary bed of the liver [16] and is a natural convective cooling term. Besides this term, forced convective heat transfer can be considered where the velocity field of the blood needs to be taken into account [17]. We assume in our study only natural convective cooling terms as in e.g. [16], [18] where the tumoral tissue is located in the capillary bed medium in the absence of large blood veins. $k$ is the thermal conductivity, and $m$ is the convective heat transfer coefficient. Metabolic heat sources can be neglected since its effect is small compared to the heat produced by $Q$. In summary, we have the following bioheat transfer equation:

$$
\rho c_{p} \frac{\partial T(\mathbf{r}, t)}{\partial t}=\nabla \cdot(k \nabla T(\mathbf{r}, t))-m\left(T(\mathbf{r}, t)-T_{b}\right)+Q(\mathbf{r})
$$

$\rho$ is the mass density and $c_{p}$ the specific heat capacity.

Due to the application of temperature, thermal damage is inflicted to the biological tissue. The so-called Arrhenius damage index $\Omega$ can mathematically be modeled by [19], [9]

$$
\frac{\partial \Omega(\mathbf{r}, t)}{\partial t}=A \exp \left[-\frac{\Delta E_{a}}{R T(\mathbf{r}, t)}\right]
$$

where $A$ is the frequency factor, $\Delta E_{a}$ is the activation energy barrier, and $R$ is the universal gas constant $(8.314 \mathrm{~J} /(\mathrm{mol} \mathrm{K}))$. The survival rate of the biological tissue can be calculated as

$$
\alpha(\mathbf{r}, t)=\exp (-\Omega(\mathbf{r}, t))
$$

and can be employed to evaluate whether tissue has been thermally damaged (necrosis for $\alpha=0)$ or not $(\alpha=1)$.

\section{B. Monopolar and bipolar RFA}

In commercial RFA devices, the applied power is usually controlled based on impedance measurements between the electrode(s) and the grounding pad [16]. Once a certain impedance threshold is exceeded, the power is shut off for $15 \mathrm{~s}$ to allow a temperature relaxation in the tissue [16]. After this period of $15 \mathrm{~s}$, the power is re-applied. In our study on the other hand, the voltage is switched on $\left(V_{\text {on }}\right)$ until the maximum temperature in the tissue reaches a certain value. As irreversible effects for the material properties start to appear at around $90^{\circ} \mathrm{C}$ [20], we limit the maximum temperature during RFA treatment to $80^{\circ} \mathrm{C}$ to avoid inaccurate modeling. Subsequently, the voltage is switched off during a fixed period $\tau$.

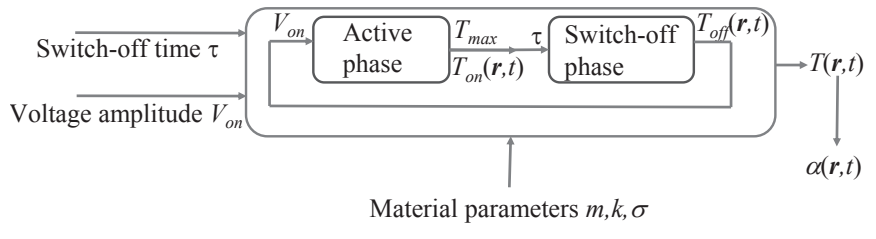

Fig. 1. Schematic illustration of the input-output relation of the computational model of radiofrequency ablation.

The complete relations between inputs and outputs for the study of monopolar and bipolar RFA are shown in figure 1. The input parameters that influence the electromagnetic source term (3) and the bioheat transfer equation (4) are $\tau$ and the voltage amplitude in the active phase $V_{o n}$. The following material parameters have a strong influence on the output as well because they are entangled in the model: the convective heat transfer coefficient $m$, the thermal conductivity $k$ and the electrical conductivity $\sigma$. The output parameters are the spatiotemporal temperature distribution $T(\mathbf{r}, t)$ and the survival rate distribution $\alpha(\mathbf{r}, t)$.

\section{Simulation setup}

We examine the possible advantages of bipolar radiofrequency ablation over monopolar radiofrequency ablation by numerically calculating pulse sequences with $V_{\text {on }}=30 \mathrm{~V}$ and $\tau=5$ s. Note that other $\tau$ values can be considered, but it remains fixed in this study in order to have the same comparison between monopolar and bipolar RFA.

Examining both calculations after an equal amount of active phases does not result in a correct comparison. Indeed, the active phases will have a different duration and at the end of $n$ pulses the total amount of time during which the voltage is applied to the needle surfaces will be different. In order to make an honest comparison between both cases, we introduce the concept of activation time. We define this activation time as the cumulative time of all active phases:

$$
\text { Activation time }(\mathrm{t})=\frac{1}{V_{\text {on }}} \int_{0}^{t}\left\|V_{n}\left(t^{\prime}\right)\right\| \mathrm{d} t^{\prime}
$$

with needle potential $V_{n}$ varying in time (on or off activation). In the bipolar RFA, both needles are activated with opposite polarities (i.e. $V_{n}$ at one needle and $-V_{n}$ at the other needle). If the activation time (7) is equal, this means that we have applied a fixed voltage $V_{\text {on }}$ during the same amount of time. In this study, we limit the total activation time to $70 \mathrm{~s}$.

We consider the following material properties of the liver tissue: $\rho=1016 \mathrm{~kg} / \mathrm{m}^{3}, c_{p}=3500 \mathrm{~J} / \mathrm{kg} / \mathrm{K}, k=0.53 \mathrm{~W} / \mathrm{m} / \mathrm{K}$ and $\sigma=0.36 \mathrm{~S} / \mathrm{m}$ following [16]. The stainless steel material properties of the needles are [21] $\rho=21500 \mathrm{~kg} / \mathrm{m}^{3}, c_{p}=132$ $\mathrm{J} / \mathrm{kg} / \mathrm{K}, k=71 \mathrm{~W} / \mathrm{m} / \mathrm{K}$ and $\sigma=4 \cdot 10^{6} \mathrm{~S} / \mathrm{m}$. The constants in the thermal damage model are [12] $A=2.984 \cdot 10^{80} \mathrm{~s}^{-1}$ and $\Delta E_{a}=5.064 \cdot 10^{5} \mathrm{~J} / \mathrm{mol}$ [9]. The convective heat transfer coefficient due to blood perfusion is $m=2.6752 \cdot 10^{4} \mathrm{~W} / \mathrm{K} / \mathrm{m}^{3}$.

The geometry consists of a sphere with diameter $8 \mathrm{~cm}$, approximating the liver. The tips of the electrodes that are placed at a certain potential have a cylindrical shape with 


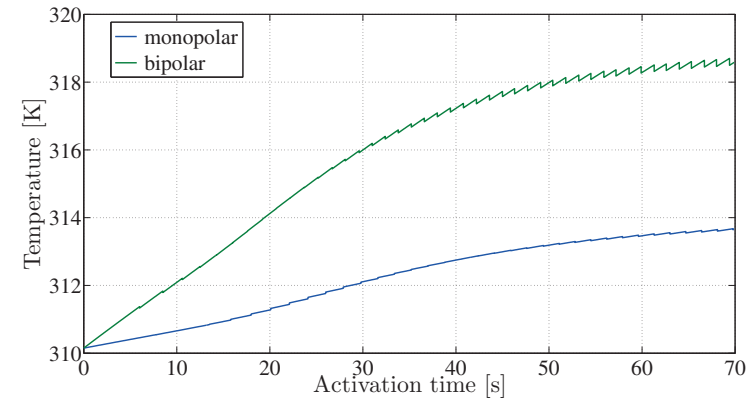

Fig. 2. Temperature at the center of the liver in function of activation time during both monopolar and bipolar RFA procedures with $V_{o n}=30 \mathrm{~V}$ and $\tau_{f}$ $=5 \mathrm{~s}$.

diameter $1 \mathrm{~mm}$ and height $2.5 \mathrm{~cm}$. In the bipolar case, we place the two needles in parallel at a distance of $2 \mathrm{~cm}$ from each other. The position of the first needle in the bipolar configuration has the same place as the needle in the monopolar configuration, i.e. they are placed at a distance of $1 \mathrm{~cm}$ from the center of the sphere. The coupled equations (3) and (4) are solved by means of the 3-dimensional finite element method. The mesh constitutes about 15000-20000 tetrahedral elements. The temperature evolution in space is calculated using the backward differentiation formula with fixed timestep of $0.1 \mathrm{~s}$. COMSOL $4.3 \mathrm{~b}$ is used to model the geometry, generate the mesh and to solve the partial differential equations.

\section{RESULTS AND DISCUSSION}

\section{A. Temperature and survival rate evolution}

Figure 2 shows the temperature at the center of the liver as function of the activation time during a monopolar and a bipolar RFA procedure. The center of the liver is the most distant point from both needle electrodes and is thus ideally suited to assess the temperature evolution in regions that normally survive RFA. A clear temperature build-up arises in both the monopolar and the bipolar case. After $70 \mathrm{~s}$ of activation time, the temperature reaches $313.7 \mathrm{~K}$ in the monopolar case (3.55 K increase), whereas the temperature in the bipolar case increases by $8.55 \mathrm{~K}$ to $318.7 \mathrm{~K}$. This figure displays the possible benefit of bipolar RFA compared to monopolar RFA. If the temperature distribution of bipolar RFA were a superposition of two individual temperature distributions of monopolar RFA, one would expect a temperature build-up that is twice as large as the individual temperature build-ups. Figure 2 however indicates that the temperature build-up is $20 \%$ larger in bipolar RFA compared to twice the temperature build-up in monopolar RFA. The reason is the spatial spread of the electric field and corresponding (3) due to the use of two needles.

The thermal damage is also quantified by means of the survival rate (6). We are especially interested in the survival rate of a circle in the central plane $z=0$ with the origin as center and with a diameter of $2 \mathrm{~cm}$. This region of interest is indicated in figure $4 \mathrm{a}$. Instead of calculating the survival rate in the center of the liver, we examine the average survival rate in this region of interest in order to include

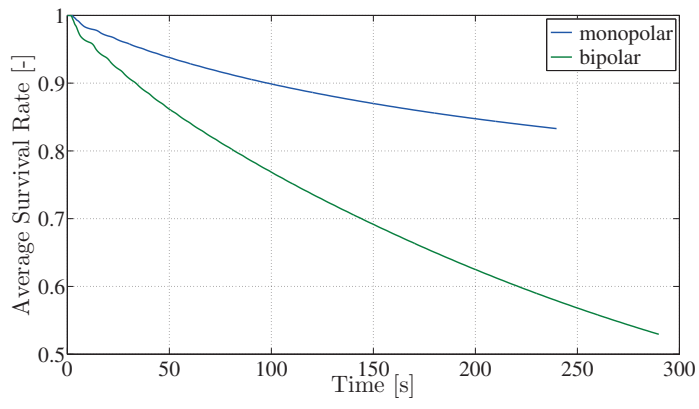

Fig. 3. Average survival rate in function of time during monopolar and bipolar RFA procedures.
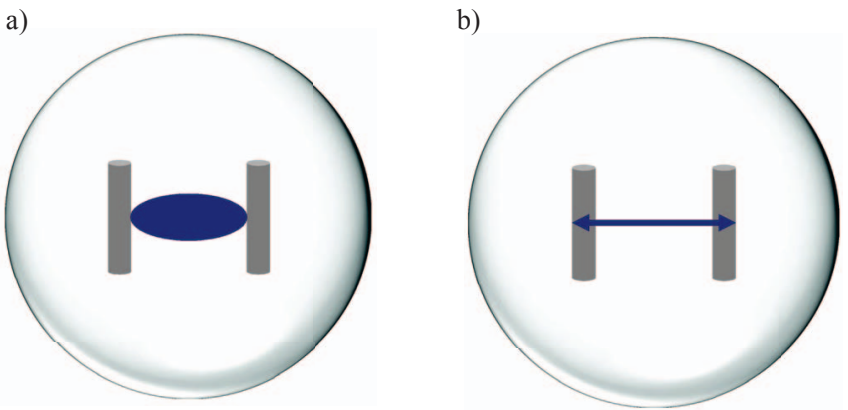

Fig. 4. (a) Graphical depiction of the region of interest (blue) when calculating the average survival rate. (b) Visualization of the line segment between both needles, which is used to study the spatial extent of the temperature distribution.

information of a larger zone. Figure 3 shows the average survival rate in function of time during both monopolar and bipolar RFA procedures. The bipolar case clearly outperforms the monopolar case. After $70 \mathrm{~s}$ of activation time, monopolar RFA is able to destroy $17 \%$ of the cells in the region of interest $(\alpha=0.83)$. The average survival rate of bipolar radiofrequency ablation on the other hand is 0.53. Again we observe that bipolar RFA destroys $38 \%$ more cells compared to twice the destruction of monopolar RFA $\left(\frac{0.47}{2 \cdot 0.17} \approx 1.38\right)$.

\section{B. Spatial extent of temperature in monopolar and bipolar $R F A$}

The spatial extent of the temperature distribution can also be examined by considering the temperature along the line segment that connects both needles (figure 4b). Figure 5 and figure 6 depict the temperature along this line segment as a function of time in the monopolar and bipolar case respectively. In these figures, the single needle in monopolar RFA is situated along the line segment at $\mathrm{x}$-coordinate $x=$ $0.55 \mathrm{~cm}$. There is another needle at $x=2.55 \mathrm{~cm}$ in the bipolar case. The center of the liver is positioned in the middle of both needles $(x=1.55 \mathrm{~cm})$. The temperature distribution is divided in temperature intervals of $5 \mathrm{~K}$ with a different colour in order to easily differentiate between the spatial extents in both cases.

In both cases, the pulses are clearly visible as the temperature steeply rises in the active phase and decreases during the switch-off phase. The zones with the highest temperature, i.e. 


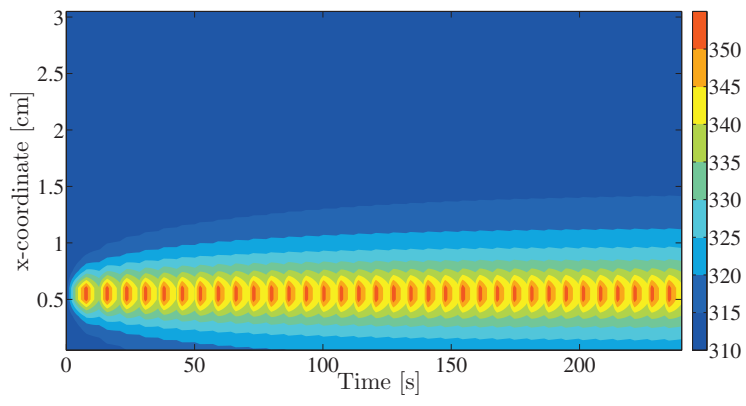

Fig. 5. Spatial extent of the temperature distribution in the monopolar case.

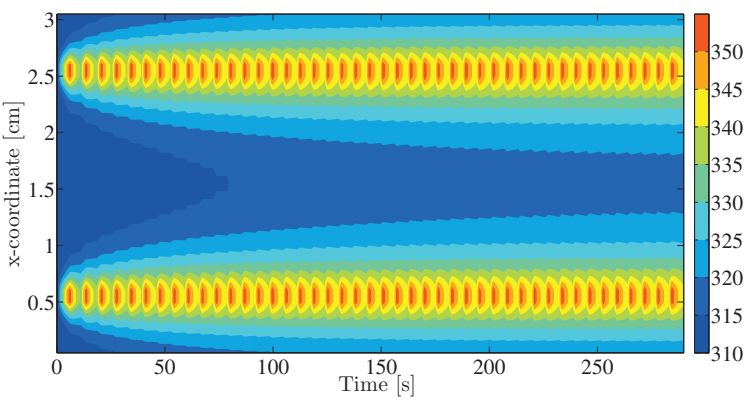

Fig. 6. Spatial extent of the temperature distribution in the bipolar case.

$T>350 \mathrm{~K}$, have a spatial extent of about $1 \mathrm{~mm}$. This spatial extent does not change during the RFA procedure. The extent of the other zones however increases with increasing time. This especially happens for the temperature interval $[315-320 \mathrm{~K}]$. After the first pulse, this zone has a size of about $1 \mathrm{~mm}$ in the monopolar case (figure 5). The size of the [315 - 320 $\mathrm{K}]$-zone increases to $3 \mathrm{~mm}$ at the end of the procedure. This indicates that the pulse sequences indeed cause an increased thermal diffusion towards distant regions. Starting from $x=$ $1.45 \mathrm{~cm}$, the temperature is however always lower than 315 $\mathrm{K}$. Consequently, this region is not thermally damaged. In the monopolar case, the thermal damage is thus limited to a cylinder around the needle electrode with a radius smaller than $1 \mathrm{~cm}$.

Figure 6 shows the spatial extent of the temperature distribution in the bipolar case. It is immediately clear that the temperature build-up is indeed larger compared to monopolar RFA. Consequently, the size of each temperature zone is larger, as well as the spatial increase of each zone with increasing time. Starting from $79 \mathrm{~s}$ for instance, the temperature in the center of the liver is always larger than $315 \mathrm{~K}$. So the intermediary zone that normally survives RFA is continuously exposed to hyperthermic temperatures between $315 \mathrm{~K}$ and $320 \mathrm{~K}$. Therefore, the thermal damage in pulsed bipolar RFA is more complete compared to monopolar RFA: the entire region between the needles is targeted. The temperature in the volume extending outside of the electrode setup however resembles the monopolar result. There is thus no benefit from any overlapping ablation zones outside of the region of interest. Consequently, the bipolar RFA configuration is ideal to completely damage tumors with a diameter of up to $3 \mathrm{~cm}$, when the needle electrodes are positioned close to the tumor boundaries (within $5 \mathrm{~mm}$ ).

\section{CONCLUSION}

This paper presents a numerical model for both monopolar and bipolar radiofrequency ablation. The computational model is based on the 3D finite element method and is able to numerically determine the spatio-temporal temperature distribution. Firstly, the benefits of bipolar radiofrequency ablation over monopolar radiofrequency ablation are illustrated. The temperature build-up in bipolar RFA is $20 \%$ larger compared to twice the build-up in monopolar RFA. Furthermore, a bipolar RFA configuration destroys 38\% more cells. The spatial extent of the temperature distribution is larger as well. The zone between the two needles is continuously exposed to at least hyperthermic temperatures in the $[315-320 \mathrm{~K}]$-interval, whereas the monopolar configuration only achieves therapeutic temperatures exceeding $315 \mathrm{~K}$ in a limited local region. Therefore, the thermal damage in pulsed bipolar radiofrequency ablation is more complete compared to monopolar radiofrequency ablation.

In the future, we will perform a detailed study of the influence of all input and material parameters of the model. Moreover, we will devote attention to study the benefit of bipolar RFA in suboptimal electrode positions. Finally, we plan ex-vivo experiments to validate our computational model.

\section{REFERENCES}

[1] S.A. Curley, F. Izzo, P. Delrio, L.M. Ellis, J. Granchi, P. Vallone, F. Fiore, S. Pignata, B. Daniele, and F. Cremona, "Radiofrequency ablation of unresectable primary and metastatic hepatic malignancies: Results in 123 patients," Annals of Surgery, vol. 230, pp. 1-8, 1999

[2] A.C.Y. Chan, R.T.P. Poon, T.T. Chueng, K.S.H. Chok, S.C. Chan, S.T. Fan, and C.M. Lo, "Survival analysis of re-section versus radiofrequency ablation for intrahepatic recurrence after hepatectomy for hepatocellular carcinoma," World Journal of Surgery, vol. 36, pp. 151-156, 2012.

[3] R. Lencioni, H-P. Allgaier, D. Cioni, M. Olschewski, P. Deibert, L. Crocetti, H. Frings, J. Laubenberger, I. Zuber, H.E. Blum, and C. Bartolozzi, "Small hepatocellular carcinoma in cirrhosis : randomized comparison of radio-frequency thermal ablation versus percutaneous ethanol injection," Radiology, vol. 229, pp. 411, 2003.

[4] S.A. Curley, "Radiofrequency ablation versus resection for resectable colorectal liver metastases: time for a randomized trial?," Annals in Surgical Oncology, vol. 15, pp. 144-157, 2007.

[5] D.A. Gervais, F.J. McGovern, B.J. Wood, S.N. Goldberg, W.S. McDougal, and P.R. Mueller, "Radio-frequency ablation of renal cell carcinoma: early clinical experience," Radiology, vol. 217, pp. 665-672, 2000.

[6] G.M. Vanderschueren, A.H. Taminiau, W.R. Obermann, and J.L. Bloem, "Osteoid osteoma: clinical results with thermocoagulation," Radiology, vol. 224, pp. 82-86, 2002.

[7] R.A. McTaggart, and D.E. Dupuy, "Thermal ablation of lung tumors," Techniques in Interventional Radiology, vol. 10, pp. 102-113, 2007.

[8] C. Chen, M. Miga, and R. Galloway, "Optimizing electrode placement using finite element models in radiofrequency ablation treatment planning,' IEEE Transactions on Biomedical Engineering, vol. 56, pp. $237-$ 245, 2009.

[9] I. dos Santos, D. Haemmerich, D. Schutt, A. Ferreira da Rocha, and L. Rax Menezes, "Probabilistic finite element analysis of radiofrequency liver ablation using the unscented transform," Physics in Medicine and Biology, vol. 54, pp. 627-640, 2009.

[10] D. Haemmerich, and D. Schutt, "RF ablation at low frequencies for targeted tumor heating: In vitro and computational modeling results," IEEE Transactions on Biomedical Engineering, vol. 58, pp. 404-410, 2011.

[11] P. Wust, B. Hildebrandt, G. Sreenivasa, B. Rau, J. Gellermann, H. Riess, R. Felix, and P.M. Schlag, "Hyperthermia in combined treatment of cancer,' Lancet Oncology, vol. 3, pp. 487-497, 2002. 
[12] S. Tungjitkusolmun, S.T. Staelin, D. Haemmerich, J.Z. Tsai, H. Cao, J.G. Webster, F.T. Lee, D.M. Mahvi, and V.R. Vorperian, "Three-dimensional finite-element analyses for radio-frequency hepatic tumor ablation," IEEE Transactions on Biomedical Engineering, vol. 49, pp. 3-9, 2002.

[13] F. Soetaert, G. Crevecoeur, L. Dupré, "Optimizing bipolar radiofrequency ablation treatment by means of pulsed currents," IEEE Engineering in Medicine and Biology Society Conference Proceedings, pp. 3745-3748, 2013.

[14] A. Gonzales-Suarez, M. Trujillo, J. Koruth, A. d'Avila, E. Berjano, "Radiofrequency cardiac ablation with catheters placed on opposing sides of the ventricular wall: Computer modelling comparing bipolar and unipolar modes," International Journal of Hyperthermia, vol. 30, pp. 372 $384,2014$.

[15] H.H. Pennes, "Analysis of tissue and arterial blood temperatures in the resting human forearm," Journal of Applied Physiology, vol. 1, pp 93$122,1948$.

[16] D. Haemmerich, and B.J. Wood, "Hepatic radiofrequency ablation at low frequencies preferentially heats tumour tissue," International Journal of Hyperthermia, vol. 22, pp. 563-574, 2006.

[17] S.Y. Choi, B.K. Kwak, and T. Seo, "Mathematical modeling of radiofrequency ablation for varicose veins," Computational and mathematical methods in medicine, Art. No. 485353, 2014.

[18] J. Jiménez-Lozano, P. Vacas-Jacques, R.R. Anderson, and W. Franco, "Selective and localized radiofrequency heating of skin and fat by controlling surface distributions of the applied voltage: analytical study," Physics in Medicine and Biology, vol. 57, pp. 7555-7578, 2012.

[19] J.A. Pearce, "Relationship between Arrhenius models of thermal damage and the CEM 43 thermal dose," Proceedings of the SPIE, vol. 7181, pp. $1-15,2009$.

[20] A. Bhattacharya, and R.L. Mahajan, "Temperature dependence of thermal conductivity of biological tissues," Physiological Measurement, vol. 24, pp. 769-83, 2003.

[21] D.J. Schutt, and D. Haemmerich, "Effects of variation in perfusion rates and of perfusion models in computational models of radio frequency tumor ablation," Medical Physics, vol. 35, pp. 3462-3470, 2008. 